\title{
Creating an equitable evidence base for quality and safety in remote antenatal care
}

\author{
Karolina Kuberska ${ }^{1}$, Francesca Dakin ${ }^{1}$, Mary Dixon-Woods ${ }^{2}$, Christine Ekechi ${ }^{3}$, and Lisa \\ Hinton $^{2}$ \\ ${ }^{1}$ University of Cambridge School of Clinical Medicine \\ ${ }^{2}$ University of Cambridge \\ ${ }^{3}$ Imperial College Healthcare NHS Trust
}

December 22, 2020

Commentary

Creating an equitable evidence base for quality and safety in remote antenatal care

Karolina Kuberska ${ }^{1}$ (PhD, https://orcid.org/0000-0002-9610-1863)

Francesca Dakin ${ }^{1}$ (MPhil, https://orcid.org/0000- 0000-0002-4105-4617)

Mary Dixon-Woods ${ }^{1}$ (DPhil; https://orcid.org/0000-0002-5915-0041)

Christine Ekechi ${ }^{2,3}$ (MD)

Lisa Hinton $^{1}$ (PhD, https://orcid.org/0000-0002-6082-3151)

${ }^{1}$ THIS Institute (The Healthcare Improvement Studies Institute), University of Cambridge, Cambridge Biomedical Campus, Clifford Allbutt Building, Cambridge CB2 0AH, UK

${ }^{2}$ Consultant Obstetrician \& Gynaecologist, Queen Charlotte's \& Chelsea Hospital, Imperial College Healthcare NHS Trust, London, UK

${ }^{3}$ Co-Chair, Race Equality Taskforce, The Royal College of Obstetricians \& Gynaecologists, UK

Correspondence to: Lisa Hinton, THIS Institute, University of Cambridge, Cambridge Biomedical Campus, Clifford Allbutt Building, Cambridge CB2 0AH, UK.

lisa.hinton@thisinstitute.cam.ac.uk; 01223331573

Shortened title: Equitable evidence base for remote antenatal care

Commentary

Creating an equitable evidence base for quality and safety in remote antenatal care

By international standards, pregnancy and childbirth are very safe in the UK. However, when poor pregnancy and birth outcomes happen, they are spread unevenly across the population. Recent reports from confidential enquiries into maternal deaths and morbidity in the UK and Ireland (MBRRACE-UK) reveal a disturbing pattern of increasing inequality.(1) Poor outcomes are associated with ethnicity and adverse socio-economic circumstances. Black women (including African, Caribbean and other Black ethnic backgrounds) are five times more likely to die as a result of complications in their pregnancy than White women.(1) For women of mixed ethnicity, the risk relative to White women is threefold, and for Asian women it is double. Women 
with multiple disadvantages, such as a mental health diagnosis or experience of domestic abuse, are also over-represented among the women who die.(1)

One of the striking features of the current COVID-19 pandemic is its amplification of these and other inequalities. Mortality rates from the coronavirus are highest for Black and Asian ethnic groups. Excess deaths are 4.3 times higher for the Black African population, 2.5 times higher for the Black Caribbean population and as much as 7.3 times higher for the other Black ethnicities than expected for this population under non-pandemic conditions.(2) Pregnancy and childbirth do not seem to escape these effects. The first study to estimate the incidence of admission to hospital with COVID-19 in pregnancy showed the highest proportion of women admitted to hospital were from Black or minority ethnic groups (56\%).(3) While the exact reasons are not yet well understood, contributing factors include a higher risk of infection, a higher risk of severe disease, or both - among others.(3)

In addressing the challenges of inequality in maternity care, a high-quality evidence-base is essential. However, a well-founded conception of what quality of evidence means is also critical. It is not enough that evidence be technically and scientifically robust, it must also be equitable and inclusive. The need for such an evidence-base is well illustrated by one of the major responses to the imperatives of the pandemic: the rapid introduction of remote care pathways. Without an inclusive body of evidence, there are real possibilities that remote care might compound the problems of marginalisation, disadvantage, and clinical risk for women in some of the most at-risk groups.

Antenatal care plays a key role in supporting women and their families during pregnancy and in improving the likelihood of optimal birth outcomes. It warrants particular scrutiny in this respect, given that access challenges are already experienced by some women of minority ethnic backgrounds and those who are socioeconomically disadvantaged.(1) An audit of referral delays in antenatal bookings, for example, highlighted inadequate care caused by language barriers and poor understanding of maternity services in the UK, and perceptions of pregnancy care services as a system of surveillance rather than support.(4) The rapid shift to remote antenatal care was an understandable - and rational - response to the imperatives of the pandemic. This included the need to minimise risks of virus transmission and the need to address the complexity associated with suspected or confirmed COVID-19 infection in pregnant women.(5) But the evidence-base for remote antenatal care remains weak. Though some studies examining use of remote technologies in antenatal care pathways have shown promising results in terms of safety and experience, $(6,7)$ they are not conclusive. Some have assessed remote monitoring of isolated components of maternity care (e.g. selfmonitored measurements of blood pressure or glucose levels), rather than the whole care pathway. $(8,9)$ Other studies have investigated hybrid antenatal care pathways that include, but are not exclusively formed of, components of remote care. (6) How easily these findings can be extrapolated into scenarios where most or all antenatal care is being provided remotely is not clear.

A perhaps less obvious problem, but one that is highly consequential for understanding and addressing inequalities, is that the participants in these studies tend not to represent groups who experience, on average, worse maternity outcomes.(1) A significant proportion of the evidence on the effectiveness and safety of remote antenatal monitoring comes from studies with homogeneous populations. Women of minority ethnic backgrounds, refugees, people experiencing homelessness, people with poor fluency in English, or those experiencing domestic abuse are rarely represented. Studies assessing satisfaction with hybrid models, for example, often include only participants who selected this care pathway when offered the choice. The participants tend to be more comfortable advocating for themselves, already have children, be white, have middle to high incomes, and be relatively highly educated.(10)

The exclusion of less privileged voices from these studies reflects a more widespread and longstanding "orthodoxy of sameness" in health research.(11) Caroline Criado Perez, in her 2019 book "Invisible Women", exposed the enduring male default bias in medical research. Clinical trials are especially prone to collecting data mostly from men and extrapolating it on the general population, which results in serious data gaps in many aspects of women's health. Evidence from maternity care research, while obviously not suffering from lack of data on women in general, may be vulnerable to similarly serious gaps when it comes to data on 
ethnicity, race and socio-economic status.

The current lacunae in the evidence for antenatal care matter: it is not safe to assume that all pregnant women have the same needs, preferences and expectations of care. Anticipating potential unintended consequences based on what is already known leaves systems better equipped both to mitigate negative impacts, and to monitor the consequences for the groups affected.(12) For example, when it comes to introducing telemedicine into antenatal care pathways, it is reasonable to make provisions for instances of technology failures (poor internet connection, insufficient mobile data), disabilities that make it difficult to use the telephone (deafness or being hard-of-hearing), or social factors (digital exclusion or experience of domestic abuse). With these perspectives missing, we simply do not have a good understanding of how well remote care can be optimised for those whose experiences are not usually included in research.

The gaps in data on race and ethnicity in health research are especially problematic for who is represented and included, and what that then means for how services are designed, for whom, and with what consequences. Addressing these gaps is hindered by the lack of clarity and understanding around the categories of race and ethnicity and how the definitions (and differences) assigned to them manifest in everyday interactions, research, and policy and practice, depending on context. For instance, in the UK, the categories of "race" (broadly defined by the symbolic colour of a person's skin or physical appearance) and "ethnicity" (reflecting a historical-cultural or national group a person may identify with) are often merged in a hybrid category of "ethnicity", which is reductionist and conceals as much as it reveals. For instance, a category such as "Black Caribbean" uses a symbolic skin colour and an ethnically non-homogenous politico-geographic region to indicate a supranational identity that limits insight into potentially highly variable help-seeking behaviours, patterns of access, and quality of care. Thus, even though we know that maternal mortality figures show five times more Black women die than their White counterparts, the Office of National Statistics (ONS) categories we have available to us do not give us the rich detail to understand why this occurs.

The broad ONS categories may obscure important ethnic differences that could illuminate our understanding of maternal mortality and morbidity within certain groups. A Black British woman, born in the UK but with a Nigerian ethnic heritage, might not encounter barriers in navigating the health system for her pregnancy. Accustomed to the UK system of healthcare, she might readily engage with antenatal care even when delivered in a remote format. In contrast, a Black Sudanese woman, recently arrived in the UK, might encounter significant barriers in accessing the care she needs. Her cultural heritage may place greater significance on the advice and support of older women in the community rather than from healthcare professionals. She would therefore have lower expectations of the antenatal care system. Both of these women are currently categorised as "Black African." Their different ethnic backgrounds, however, potentially influence their belief systems and behaviours, and may affect their individual risks of poor maternal outcomes.

Problematic as racial and ethnic identities are, capturing these categories as research data is important. Race and ethnicity may be social constructs, but they are real in their consequences, powerfully impacting healthcare access and outcomes in profound ways.(1) Understanding the consequences of race and ethnicity for women accessing healthcare, coupled with a recognition of the significance of socio-economic determinants of health, can help create pathways that address patients' needs in more nuanced and socio-culturally sensitive ways. Without granular data on who is at greatest risk of severe maternal morbidity and mortality, we are likely to entrench racialised stereotypes without reducing inequalities. It is important to go beyond simple dichotomies (e.g. that imply White and "Other"): we must ask ourselves challenging questions about how to ensure authentic inclusion, the definitions we are using to construct the world we are seeking to describe, and what we are seeking to improve for whom.

Given that COVID-19 does not impact all population subgroups in the same way, an understanding of what good remote antenatal care looks like is urgently needed to help shape pathways that offer appropriate support for every pregnant woman. At the same time, as sound evaluation of models of antenatal care which had to be introduced very rapidly - is urgently needed, such evaluation must be highly attentive to the diversity of experiences and needs. Remote care may be safe for large sections of the pregnant population, but may also create unintended barriers for some. Creating equitable antenatal care pathways requires 
intentional and sustained effort not only to prevent new harms but also to reduce existing institutional racism and structural inequalities in healthcare. Once the pandemic passes, antenatal care is unlikely to return to pre-pandemic models in its entirety. We have an opportunity to capture what has worked well and to mobilise that learning for the benefit of pregnant women. But it is also vital that the evidence we create is equitable.

\section{Acknowledgements}

We thank Professor Graham Martin for commenting on earlier versions of the draft.

\section{Disclosure of interest}

KK, FD, MDW and LH declare no competing interests. CE is the Director of Early Pregnancy Plus, Indak LTD, Board Trustee of The Eve Appeal Charity, Advisory Board Member of Baby Lifeline Charity, she has received speaking fees from Harvard Medical School, The Wing, Visa, and Diageo, outside the submitted work.

\section{Contribution to authorship}

KK and LH developed the idea for and led the drafting of the commentary. FD assisted in research and contributed to the drafting of the manuscript. CE and MDW contributed to the drafting of the manuscript.

\section{Details of ethics approval}

Ethics approval was not required for this Commentary.

\section{Funding}

KK, FD, MDW and LH are supported by the Health Foundation's grant to the University of Cambridge for The Healthcare Improvement Studies (THIS) Institute. THIS Institute is supported by the Health Foundation - an independent charity committed to bringing about better health and health care for people in the UK.

1. Knight M, Bunch K, Tuffnell D, Shakespeare J, Kotnis R, Kenyon S, et al. Saving Lives, Improving Mothers' Care Lessons learned to inform maternity care from the UK and Ireland Confidential Enquiries into Maternal Deaths and Morbidity 2015-17. MBRRACE-UK; 2019.

2. Razaq A, Harrison D, Karunanithi S, Barr B, Asaria M, Routen A, et al. BAME COVID-19 Deaths - what do we know? Rapid data and evidence review: 'hidden in plain sight'. Oxford: The Centre for Evidence-Based Research; 2020.

3. Knight M, Bunch K, Vousden N, Morris E, Simpson N, Gale C, et al. Characteristics and outcomes of pregnant women admitted to hospital with confirmed SARS-CoV-2 infection in UK: national population based cohort study. BMJ. 2020;369:m2107.

4. McDonald H, Moren C, Scarlett J. Health inequalities in timely antenatal care: audit of pre- and postreferral delays in antenatal bookings in London 2015-16. J Public Health (Oxf). 2020.

5. Knight M, Bunch K, Cairns A, al. e. Saving Lives, Improving Mothers' Care Rapid Report: Learning from SARS-CoV-2-related and associated maternal deaths in the UK March - May 2020. Oxford: National Perinatal Epidemiology Unit, University of Oxford; 2020. Report No.: 978-0-9931267-8-9.

6. Alves DS, Times VC, da Silva EMA, Melo PSA, Novaes MA. Advances in obstetric telemonitoring: a systematic review. Int J Med Inform. 2020;134:104004.

7. van den Heuvel JF, Groenhof TK, Veerbeek JH, van Solinge WW, Lely AT, Franx A, et al. eHealth as the Next-Generation Perinatal Care: An Overview of the Literature. J Med Internet Res. 2018;20(6):e202.

8. Hinton L, Tucker KL, Greenfield SM, Hodgkinson JA, Mackillop L, McCourt C, et al. Blood pressure self-monitoring in pregnancy (BuMP) feasibility study; a qualitative analysis of women's experiences of 
self-monitoring. BMC Pregnancy Childbirth. 2017;17(1):427.

9. Ming WK, Mackillop LH, Farmer AJ, Loerup L, Bartlett K, Levy JC, et al. Telemedicine Technologies for Diabetes in Pregnancy: A Systematic Review and Meta-Analysis. J Med Internet Res. 2016;18(11):e290.

10. Butler Tobah YS, LeBlanc A, Branda ME, Inselman JW, Morris MA, Ridgeway JL, et al. Randomized comparison of a reduced-visit prenatal care model enhanced with remote monitoring. Am J Obstet Gynecol. 2019;221(6):638 e1- e8.

11. Healy B. Challenging Sameness: Women in Clinical Trials. Bethesda, MD: National Institutes of Health, Office of Research on Women's Health; 2003 January 6-9.

12. Bonell C, Jamal F, Melendez-Torres GJ, Cummins S. 'Dark logic': theorising the harmful consequences of public health interventions. J Epidemiol Community Health. 2015;69(1):95-8. 